\title{
Reactive compensator synthesis in time-domain
}

\author{
M. SIWCZYŃSKI and M. JARACZEWSKI* \\ Department of Eletrical and Computer Engineering, Cracow University of Technology, 24 Warszawska St., 31-425 Cracow, Poland
}

\begin{abstract}
The source reactive-current compensation is crucial in the energy transmission efficiency. The compensator design in a frequencydomain has already been widely discussed and examined. This paper presents results of a study on how to design reactive compensators in a time-domain. It is the first time the reactive compensator has been designed in a time domain. The example of a compensator is presented.
\end{abstract}

Key words: power and energy theory, reactive power filters, reactive current, optimization, harmonic filter design.

\section{Introduction}

This article is a discussion on issue raised in the article of L.S. Czarnecki [1] where the author considers if it is possible to make a current decomposition into active, reactive and unbalanced current in a time domain and basing on it to build reactance compensators. The current decomposition in a time domain was presented in the previous articles [2, 3] and in this article the reactive compensators design in a time-domain is presented.

\section{Reactive current compensation in time-domain}

In the article [3], there was shown that a source-load current can be decomposed into active and reactive current in 's' domain i.e. for the Laplace transform of signals. Reactive current can be compensated with the reactive compensator. The source-load current decomposition is given below:

$$
I(s)=G^{o}(s) E(s)+B^{o}(s) E(s)=I_{G}^{o}(s)+I_{B}^{o}(s),
$$

where

$$
\begin{gathered}
G^{o}(s)=\frac{1}{2}\left(Y^{o}(s)+Y^{o}(-s)\right), \\
B^{o}(s)=\frac{1}{2}\left(Y^{o}(s)_{Y}^{o}(-s)\right)
\end{gathered}
$$

stand for the active and reactive parts of load admittance operator $Y^{o}(s)$.

The equivalent decomposition (1) in time-domain is the $T$-periodic convolution:

$$
i(t)=\left(g^{o}(t)+b^{o}(t)\right) \otimes e(t 0
$$

where $g^{o}(t), b^{o}(t)$ stands for T-periodic impulse response of a load admittance active and reactive part given from the inverse relations [3]

$$
\begin{aligned}
& \frac{1}{\sigma+s} \longrightarrow \frac{e^{-\sigma t}}{1-e^{-\sigma t}}, \\
& \frac{1}{\sigma-s} \longrightarrow \frac{e^{\sigma(t-T)}}{1-e^{-\sigma T}},
\end{aligned}
$$

where $t \in[0, T), \operatorname{Re}(\sigma)>0, T-$ time period.
Since $b^{k}(t)$ stands for $T$-periodic impulse response of a compensator admittance reactive part, thus the reactive current balance in time-domain is:

$$
b^{k}(t)+b^{o}(t)=0 .
$$

We assume that the compensator is composed of almost lossless elementary branches connected in parallel as well as the load (Fig. 1).

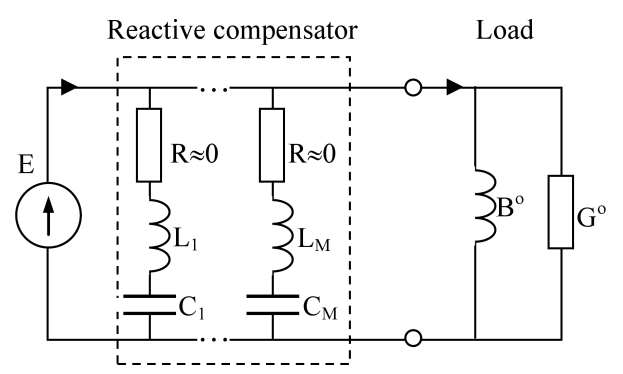

Fig. 1. The zero impedance source, load and almost lossless compensator connected in parallel

Reactive part of elementary compensator branch is:

$$
B(s)=\frac{d(-a)}{a+s}+\frac{d\left(-a^{*}\right)}{a^{*}+s}+\frac{d(a)}{a-s}+\frac{d\left(a^{*}\right)}{a^{*}-s}=\frac{L(s)}{M(s)},
$$

where $L(s), M(s)$ - odd and even polynomials.

Residues are given by formulas:

$$
\begin{gathered}
d(-a)=\left.[B(s)(a+s)]\right|_{s=-a}=\frac{L(-a)}{M^{\prime}(-a)}=\frac{L(s)}{M^{\prime}(a)} \equiv d, \\
d(a)=\left.[B(s)(a-s)]\right|_{s=a}=-\frac{L(a)}{M^{\prime}(a)}=-d, \\
d\left(-a^{*}\right)=\left.\left[B(s)\left(a^{*}+s\right)\right]\right|_{s=-a^{*}}=\frac{L\left(-a^{*}\right)}{M^{\prime}\left(-a^{*}\right)} \\
=\frac{L\left(a^{*}\right)}{M^{\prime}\left(a^{*}\right)}=\left[\frac{L(a)}{M^{\prime}(a)}\right]^{*}=d^{*} \\
d\left(a^{*}\right)=\left.\left[B(s)\left(a^{*}-s\right)\right]\right|_{s=a^{*}}=-\frac{L\left(a^{*}\right)}{M^{\prime}\left(a^{*}\right)} \\
=\left[\frac{L(a)}{M^{\prime}(a)}\right]^{*}=-d^{*},
\end{gathered}
$$

*e-mail: e-3pk.edu.pl 
where $M^{\prime}(s)$ is the derivative of $M(s)$ with respect to $s, d-$ real number.

Thus (7) reduces to

$$
B(s)=d\left(\frac{1}{a+s}-\frac{1}{a-s}\right)+d^{*}\left(\frac{1}{a^{*}+s}-\frac{1}{a^{*}-s}\right)
$$

and under (5)

$$
\begin{aligned}
b^{k}(t) & =d \frac{e^{-a t}-e^{a(t-T)}}{1-e^{-a T}}+d^{*} \frac{e^{-a^{*} t}-e^{a^{*}(t-T)}}{1-e^{-a^{*} T}} \\
& =-2 \operatorname{Re}\left(\frac{\operatorname{sh}\left(A\left(\frac{t}{T}-\frac{1}{2}\right)\right)}{\operatorname{sh}\left(\frac{A}{2}\right)}\right),
\end{aligned}
$$

where $A=a T=\alpha+j \beta$.

From the trigonometric identity

$$
\operatorname{sh}(\alpha+j \beta)=\operatorname{sh}(\alpha) \cos (\beta)+j \operatorname{ch}(\alpha) \sin (\beta)
$$

results that

$$
\begin{aligned}
b^{k}(t) & =-2 d \operatorname{Re}\left(\frac{\operatorname{sh}\left(\alpha\left(\frac{t}{T}-\frac{1}{2}\right)\right) \cos \left(\beta\left(\frac{t}{T}-\frac{1}{2}\right)\right)}{\operatorname{sh}\left(\frac{\alpha}{2}\right) \cos \left(\frac{\beta}{2}\right)+j \operatorname{ch}\left(\frac{\alpha}{2}\right) \sin \left(\frac{\beta}{2}\right)}\right. \\
& \left.+j \frac{\operatorname{ch}\left(\alpha\left(\frac{t}{T}-\frac{1}{2}\right)\right) \sin \left(\beta\left(\frac{t}{T}-\frac{1}{2}\right)\right)}{\operatorname{sh}\left(\frac{\alpha}{2}\right) \cos \left(\frac{\beta}{2}\right)+j \operatorname{ch}\left(\frac{\alpha}{2}\right) \sin \left(\frac{\beta}{2}\right)}\right)
\end{aligned}
$$

and for $\alpha \rightarrow 0$

$$
\begin{aligned}
b^{k}(t) & =-2 d \frac{\operatorname{ch}\left(\alpha\left(\frac{t}{T}-\frac{1}{2}\right)\right) \sin \left(\beta\left(\frac{t}{T}-\frac{1}{2}\right)\right)}{\operatorname{ch}\left(\frac{\alpha}{2}\right) \sin \left(\frac{\beta}{2}\right)} \\
& =-2 d \frac{\sin \left(\beta\left(\frac{t}{T}-\frac{1}{2}\right)\right)}{\sin \left(\frac{\beta}{2}\right)} t \in[0, T) .
\end{aligned}
$$

If it is the case of RLC compensator elementary branch then

$$
Y^{k}(s)=\frac{1}{L} \frac{s}{\omega_{o}^{2}+2 \epsilon s+s^{2}} ; \quad \omega_{o}=\frac{1}{\sqrt{L C}}, \quad \epsilon=\frac{R}{2 L} .
$$

Its reactive part is

$$
\begin{gathered}
B^{k}(s)=\frac{1}{2 L}\left(\frac{s}{\omega_{o}^{2}+2 \epsilon s+s^{2}}+\frac{s}{\omega_{o}^{2}-2 \epsilon s+s^{2}}\right) \\
=\frac{1}{2 L}\left(\frac{s}{(a+s)\left(a^{*}+s\right)}+\frac{s}{(a-s)\left(a^{*}-s\right)}\right),
\end{gathered}
$$

where $a=\epsilon+j \omega_{o} \approx j \omega_{o}$

$$
\beta=\omega_{o} T=2 \pi \sqrt{\frac{X_{C}}{X_{L}}}
$$

$X_{C}=\frac{T}{1 \pi C}, X_{L}=\frac{2 \pi L}{T}-$ capacitive and inductive reactance for the main frequency $f=1 / T$.
Residue for $B^{k}(s)$ can be calculated as

$$
d=\left.\left[B^{k}(s)(a+s)\right]\right|_{\left(\begin{array}{c}
s=-a \\
\epsilon=0
\end{array}\right)}=\frac{1}{2 L} .
$$

Thus the time depended representation of reactive part of the elementary RLC branch (without $R$ ) has the form

$$
b^{k}(t)=-\frac{1}{L} \frac{\sin \left(2 \pi \sqrt{\frac{X_{C}}{X_{L}}}\left(\frac{t}{T}-\frac{1}{2}\right)\right)}{\sin \left(\pi \sqrt{\frac{X_{C}}{X_{L}}}\right)} ; \frac{t}{T} \in[0, T] .
$$

In such a way we arrive to the compensatory set of Eqs. (6) in a new form

$$
\sum_{m=1}^{M} \frac{1}{L_{m}} \frac{\sin \left(2 \pi w_{m}\left(\frac{t}{T}-\frac{1}{2}\right)\right)}{\sin \left(\pi w_{m}\right)}=b^{o}\left(\frac{t}{T}\right)
$$

where $w_{m}=\sqrt{\left(\frac{X_{C}}{X_{L}}\right)}=\frac{\omega_{0 n}}{\left(\frac{2 \pi}{T}\right)}, m$ - number of elementary branch, $M$ - total number of compensator branches, $\omega_{o m}$ - resonance frequency of $m$-th branch.

Later in the article it is assumed that the reactive part of load $Y^{o}(s)$ has only real poles, so the load is not an oscillator circuit in contrast to the compensator.

For the single pole load

$$
B^{o}(s)=b\left(\frac{1}{a+s}-\frac{1}{a-s}\right) \quad a>0
$$

thus its invert transform is

$$
b^{o}\left(\frac{t}{T}\right)=b \frac{e^{-a t}-e^{a(t-T)}}{1-e^{-a T}}=-b \frac{\operatorname{sh}\left(A\left(\frac{t}{t}-\frac{1}{2}\right)\right)}{\operatorname{sh}\left(\frac{A}{2}\right)},
$$

where $A=a T, t / T \in[0, T)$.

The coefficient $b$ can be both positive and negative what is shown below
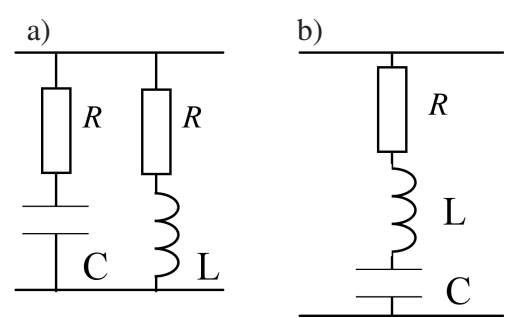

Fig. 2. Examples of the load a) RRLC b) RLC

For the RRLC load (see Fig. 2a) the admittance operator is

$$
\begin{gathered}
Y^{o}(s)=\frac{1}{R+s L}+\frac{1}{R+\frac{1}{s C}} \\
=2 b_{L} \frac{1}{a_{L}+s}-2 b \frac{1}{a_{C}+s}+\frac{1}{R},
\end{gathered}
$$

Bull. Pol. Ac.: Tech. 60(1) 2012 
where

$$
\begin{gathered}
a_{L}=\frac{R}{L}, \quad b_{C}=\frac{1}{R C}, \\
b_{L}=\frac{1}{2 L}, \quad b_{C}=\frac{a_{C}}{2 R}=\frac{1}{2 R^{2} C} .
\end{gathered}
$$

Then the reactive part of $Y_{o}(s)$ is

$$
\begin{gathered}
B^{o}(s)=b_{L}\left(\frac{1}{a_{L}+s}-\frac{1}{a_{L}-s}\right) \\
-b_{C}\left(\frac{1}{a_{C}+s}-\frac{1}{a_{C}-s}\right)
\end{gathered}
$$

and its $T$-periodic inverse transform is

$$
\begin{gathered}
b^{o}\left(\frac{t}{T}\right)=-b_{L} \frac{\operatorname{sh}\left(A_{L}\left(\frac{t}{T}-\frac{1}{2}\right)\right)}{\operatorname{sh}\left(\frac{A_{L}}{2}\right)} \\
+b_{C} \frac{\operatorname{sh}\left(A_{C}\left(\frac{t}{T}-\frac{1}{2}\right)\right)}{\operatorname{sh}\left(\frac{A_{C}}{2}\right)}
\end{gathered}
$$

where $A_{L}=a_{L} T, A_{C}=a_{C} T$.

Whereas for the load shown in Fig. 2b:

$$
Y^{o}(s)=\frac{1}{\frac{1}{s C}+R+s L}=\frac{1}{L} \frac{s}{\omega_{0}^{2}+2 \alpha s+s^{2}},
$$

where $\omega_{o}=\frac{1}{\sqrt{L C}}, \alpha=\frac{R}{2 L}$.

For the positive poles condition $\alpha^{2}-\omega^{2}>0$.

$Y^{o}(s)$ takes form

$$
Y^{o}(s)=\frac{1}{L} \frac{s}{\left(a_{1}+s\right)\left(a_{2}+s\right)}=2\left(\frac{b_{1}}{a_{1}+s}+\frac{b_{2}}{a_{2}+s}\right),
$$

where $a_{1}+\alpha+\sqrt{\alpha^{2}-\omega_{0}^{2}}, a_{2}=\alpha-\sqrt{\alpha^{2}-\omega_{0}^{2}}$.

And the coefficients $b_{1}, b_{2}$ are then

$$
\begin{aligned}
& b_{1}=\left.\frac{1}{2}\left[Y(s)\left(a_{1}+s\right)\right]\right|_{s=-a_{1}}=\frac{1}{2 L} \frac{a_{1}}{a_{1}-a_{2}}, \\
& b_{2}=\left.\frac{1}{2}\left[Y(s)\left(a_{2}+s\right)\right]\right|_{s=-a_{2}}=\frac{1}{2 L} \frac{a_{2}}{a_{1}-a_{2}},
\end{aligned}
$$

or

$$
\begin{aligned}
& b_{1}=\frac{1}{4 L} \frac{\alpha+\sqrt{\alpha^{2}-\omega_{0}^{2}}}{\sqrt{\alpha^{2}-\omega_{0}^{2}}}>0, \\
& b_{2}=-\frac{1}{4 L} \frac{\alpha-\sqrt{\alpha^{2}-\omega_{0}^{2}}}{\sqrt{\alpha^{2}-\omega_{0}^{2}}}<0,
\end{aligned}
$$

thus

$$
\begin{gathered}
B^{o}(s)=b_{1}\left(\frac{1}{a_{1}+s}-\frac{1}{a_{1}-s}\right) \\
+b_{2}\left(\frac{1}{a_{2}+s}-\frac{1}{a_{2}-s}\right) .
\end{gathered}
$$

a)
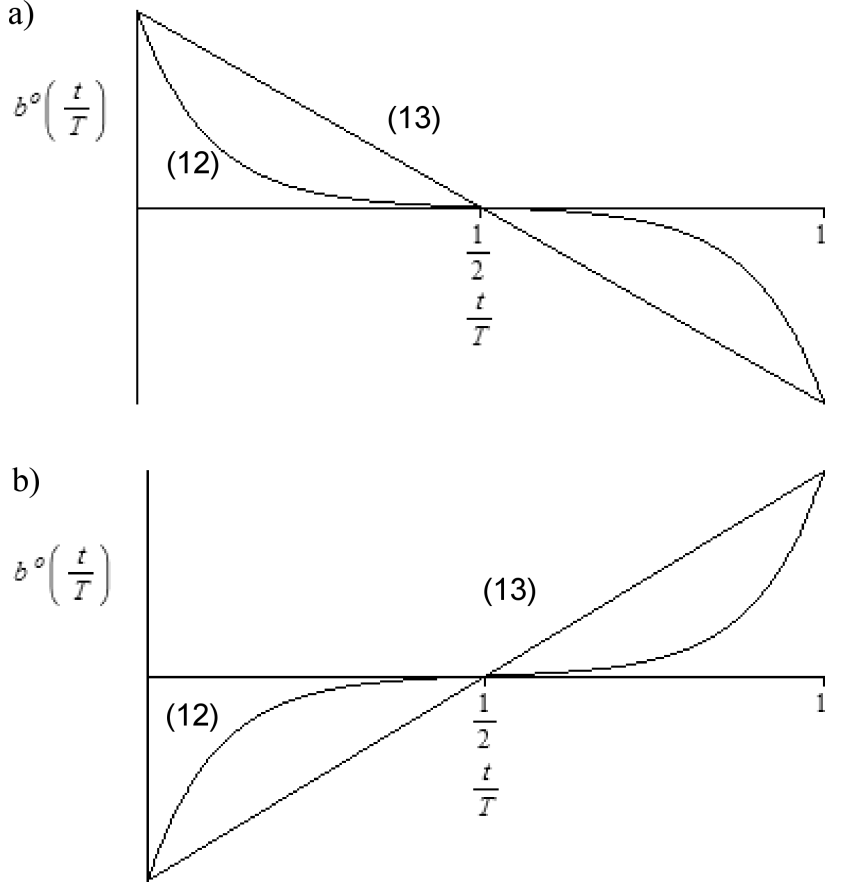

Fig. 3. T-periodic single pole reactive operator in time-domain for a) $b>0$, b) $b<0$

The reactive functions $b^{o}\left(\frac{t}{T}\right)$ (12) are shown in Fig. 3. It is necessary to distinguish the two cases when $b>0$ and $b<0$. When $A / 2 \rightarrow 0$ the curves become straight lines.

$$
b^{o}\left(\frac{t}{T}\right)=-2 b\left(\frac{t}{T}-\frac{1}{2}\right), \quad \frac{t}{T} \in[0,1)
$$

because $\operatorname{sh}(x) \rightarrow 0$ for $x \rightarrow 0$.

The solution of (11), for the unknowns $L_{m}$ and $C_{m}$, can be find with optimization method. The (11) can be then rewritten in the following form

$$
\sum_{m=1}^{M} D_{m} \sin \left(2 \pi w_{m}\left(\frac{t}{T}-\frac{1}{2}\right)\right)=b^{o}\left(\frac{t}{T}\right)
$$

where

$$
D_{m}=\frac{1}{L_{m} \sin \left(\pi w_{m}\right)} ; \quad w_{m}=\sqrt{\left(\frac{X_{C}}{X_{L}}\right)_{m}} .
$$

The relative frequencies of LC compensator branches have to meet the condition

$$
\sin \left(\pi w_{m}\right) \neq 0
$$

thus

$$
w_{m} \neq p ; \quad p-\text { even number. }
$$

The set of Eqs. (14) can be solved for $D_{m}$ by minimizing

$$
\delta^{2} \rightarrow \min ,
$$


where

$$
\begin{gathered}
\delta_{0}^{2}=\int_{0=1}^{1}\left(\sum_{m=}^{M} D_{m} \sin \left(2 \pi w_{m}\left(\frac{t}{T}-\frac{1}{2}\right)\right)\right. \\
\left.-b^{o}\left(\frac{t}{T}\right)\right)^{2} d\left(\frac{t}{T}\right)
\end{gathered}
$$

or after few simple transformations it can be rewritten as

$$
\begin{gathered}
\delta^{2}=\int_{0}^{1} b^{o}\left(\frac{t}{T}\right)^{2} d\left(\frac{t}{T}\right) \\
-2 \sum_{m=1}^{M} D_{m} \int_{0}^{1} b^{o}\left(\frac{t}{T}\right) \sin \left(2 \pi w_{m}\left(\frac{t}{T}-\frac{1}{2}\right)\right) d\left(\frac{t}{T}\right) \\
+\sum_{m=1}^{M} \sum_{n=1}^{N} \frac{\sin \left(\pi\left(w_{m}-w_{n}\right)\right)}{2 \pi\left(w_{m}-w_{n}\right)} D_{m} D_{n} \\
-\sum_{m=1}^{M} \sum_{n=1}^{N} \frac{\sin \left(\pi\left(w_{m}+w_{n}\right)\right)}{2 \pi\left(w_{m}+w_{n}\right)} D_{m} D_{n} \rightarrow \min .
\end{gathered}
$$

After equating to zero the appropriate partial derivatives $\frac{\partial}{\partial D_{m}}$ and assuming that

$$
w_{p}=p+\alpha, \quad p=1,2,3, \ldots ; \quad 0<\alpha<1,
$$

we get necessary minimum condition in form of the set of equations for $\frac{1}{L_{p}}$

$$
\begin{gathered}
\frac{1}{L_{p}}-\sum_{m=1}^{M} \frac{\sin (2 \pi \alpha)}{\pi(m+p+2 \alpha)} \frac{1}{L_{m}} \\
=\left(\sin \left(\pi w_{p}\right)\right) \int_{0}^{1} b^{o}\left(\frac{t}{T}\right) \sin \left(2 \pi w_{p}\left(\frac{t}{T}-\frac{1}{2}\right)\right) d\left(\frac{t}{T}\right) .
\end{gathered}
$$

In particular case of the one pole load, for which $b^{o}\left(\frac{t}{T}\right)$ is given by (12), Eq. (18) gets form

$$
\begin{gathered}
\frac{1}{L_{p}}-\sum_{m=1}^{M} \frac{\sin (2 \pi \alpha)}{\pi(m+p+2 \alpha)} \frac{1}{L_{m}}=\frac{b}{\left(\frac{A}{2}\right)^{2}+\left(\pi w_{p}\right)^{2}} \\
\cdot\left(\pi w_{p} \sin (2 \pi \alpha)-2 \frac{A}{2} \operatorname{cth}\left(\frac{A}{2}\right) \sin (\pi \alpha)^{2}\right)
\end{gathered}
$$

or after separating the diagonal (dominating) element we get

$$
\begin{gathered}
\left(1-\frac{\sin (2 \pi \alpha)}{2 \pi(p+\alpha)}\right) \frac{1}{L_{p}} \\
-\sum_{m \neq p} \frac{\sin (2 \pi \alpha)}{\pi(m+p+2 \alpha)} \frac{1}{L_{m}}=\frac{b}{\left(\frac{A}{2}\right)^{2}+\left(\pi w_{p}\right)^{2}} \\
.\left(\pi w_{p} \sin (2 \pi \alpha)-2 \frac{A}{2} \operatorname{cth}\left(\frac{A}{2}\right) \sin (\pi \alpha)^{2}\right) .
\end{gathered}
$$

The offset $\alpha$ in (17) must be less then 0.5 as to assure $L_{p}$ positive.

\section{Comparison with compensator design in frequency-domain}

The comparison of time-domain compensator design method to the frequency-domain one will be shown below. The frequency-domain approach is a well known method (see M. Pasko [4-6]).

The counterpart of (6) in frequency-domain is

$$
B_{n}^{k}+B_{n}^{o}=0
$$

where $B_{n}^{k}, B_{n}^{o}$ - frequency response of compensator and load susceptance, $n=1,2,3, \ldots-$ harmonic number.

For the elementary compensator branch ( $L C$ in series)

$$
Y^{k}(s)=\frac{1}{s L+\frac{1}{s C}}=\frac{2 \pi}{X_{L}} \frac{s T}{(s T)^{2}+(2 \pi)^{2} \frac{X_{C}}{X_{L}}} .
$$

Substituting $s T=\frac{j n 2 \pi}{T} T=j 2 \pi n$ we get the frequency response of the branch elementary susceptance

$$
B_{n}=-\frac{n}{n^{2}-\frac{X_{C}}{X_{L}}} \frac{1}{X_{L}} .
$$

Thus the formula of reactive source current compensation (22) takes form

$$
\sum_{m=1}^{M} \frac{n}{n^{2}-w_{m}^{2}} \frac{1}{L_{m}}=\frac{2 \pi}{T} B_{n}^{o} .
$$

The Eq. (23) is the counterpart of (11) transformed to optimization task (18).

As to perform a comparative analysis of (11) and (23) it must be examined the susceptance relation between $b^{o}\left(\frac{t}{T}\right)$ and its transform $B_{n}^{o}$ (in frequency-domain). It can be proved that

$$
B_{n}^{o}=-T \int_{0}^{1} b^{o}\left(\frac{t}{T}\right) \sin \left(2 \pi n \frac{t}{T}\right) d\left(\frac{t}{T}\right) .
$$

Then in the particular case of the $R L$ in series load we get

$$
\begin{aligned}
& B_{n}^{o}=\operatorname{Im}\left(Y^{o}\left(j \frac{2 \pi}{T} n\right)\right) \\
& =\operatorname{Im}\left(\frac{T}{L} \frac{A-j 2 \pi n}{A^{2}+(2 \pi n)^{2}}\right) \\
& =-\frac{T}{s L} \frac{\pi n}{\left(\frac{A}{2}\right)^{2}+(\pi n)^{2}},
\end{aligned}
$$

where $n$-integer. 
Certainly we can integrate Eq. (11) the same way as

$$
\begin{gathered}
-T \int_{0}^{1} \sum_{m=1}^{M} \frac{\sin \left(2 \pi w_{m}\left(\frac{t}{T}-\frac{1}{2}\right)\right)}{L_{m} \sin \left(\pi w_{m}\right)} \sin \left(2 \pi n \frac{t}{T}\right) d\left(\frac{t}{T}\right) \\
=-T \int_{0}^{1} b^{o}\left(\frac{t}{T}\right) \sin \left(2 \pi n \frac{t}{T}\right) d\left(\frac{t}{T}\right)
\end{gathered}
$$

as to get (see (23))

$$
\frac{T}{2 \pi} \sum_{m=1}^{M} \frac{1}{L_{m}} \frac{n}{\left(n^{2}-w_{m}^{2}\right)}=-\frac{X_{L} n}{R^{2}+X_{L}^{2} n^{2}}=B_{n}^{o} .
$$

The relation between time samples $p \tau$ and frequency samples $n 2 \pi / T$ is expressed in discrete time integration formula (Euler method) (see (24))

$$
\begin{gathered}
B_{n}^{k}+B_{n}^{o} \\
=\left(\sum_{p=1} N\left(b^{k}(p \tau)+b^{o}(p \tau)\right) \sin \left(n \frac{2 \pi}{T} p \tau\right)\right),
\end{gathered}
$$

where $\tau=T / N$.

If

$$
b^{k}(p \tau)+b^{o}(p \tau)=0 \quad \text { then } \quad B_{n}^{k}+B_{n}^{o}=0 .
$$

This is true only for low frequencies because it is discrete time system now.

For Euler integral method the mapping formula is

$$
s=\frac{1}{\tau}\left(1-z^{-1}\right) \quad \text { where } \quad z=e^{j \omega \tau},
$$

which ends up with

$$
s=\sigma+j \Omega=\frac{1}{\tau}(1-\cos (\omega \tau)+j \sin (\omega \tau)) .
$$

Then $\Omega \approx \omega$ and $\sigma=0$ for only small frequencies (see Fig. 4)

The relation (28) reduces the number of compensated harmonics to less then $N / 4$. As to expand the range of compensated harmonics we have to increase $N$ (time samples). Together with increase $N$ it must by increased $M$ (number of compensator branches ) because as to meet Eq. (27)

$$
b^{k}(p \tau)+b^{o}(p \tau)=0
$$

in $N$ points we need resonance compensator frequency no less then $\frac{N}{2} \frac{2 \pi}{T}$ (see Fig. 5, 6, the crossing points of $b^{o}(t)$ and $\left.-b^{k}(t)\right)$. This results that maximum $w_{p}=\frac{N}{2}$.

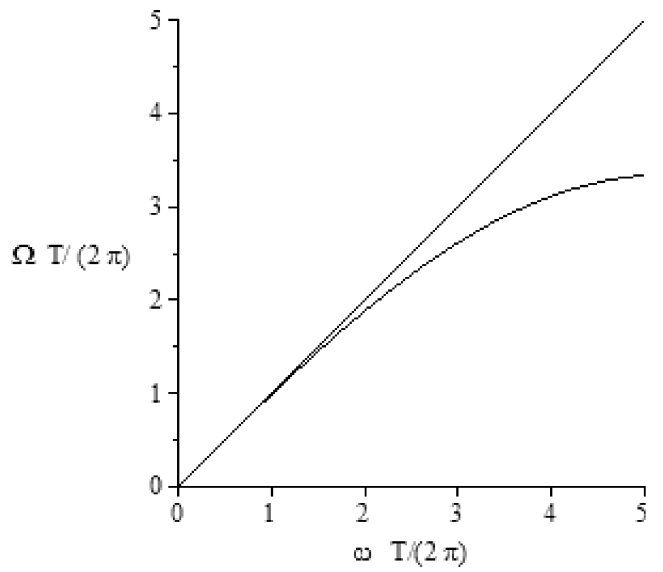

Fig. 4. Relation between $\Omega$ and $\omega$ for Euler's integral method for $N=21$ time samples a period

Thus if we wish to compensate all harmonics from 1 to $k$ we need $M=N / 2=2 k$ compensator branches.

\section{Calculation example}

Let consider the RL in series load for which: $P=500[\mathrm{~W}]$, $T=0.02[\mathrm{~s}], \omega=314[\mathrm{rad} / \mathrm{s}], A=T / \tau_{L}=10, L_{o}=25.7$ [mH] and $N=21, M=10, \tau_{L}$ - time-constant of load.

Effective compensation is up to 5 -th harmonic. lem.

Time samples are shifted by $\tau / 2$ due to a singularity prob-
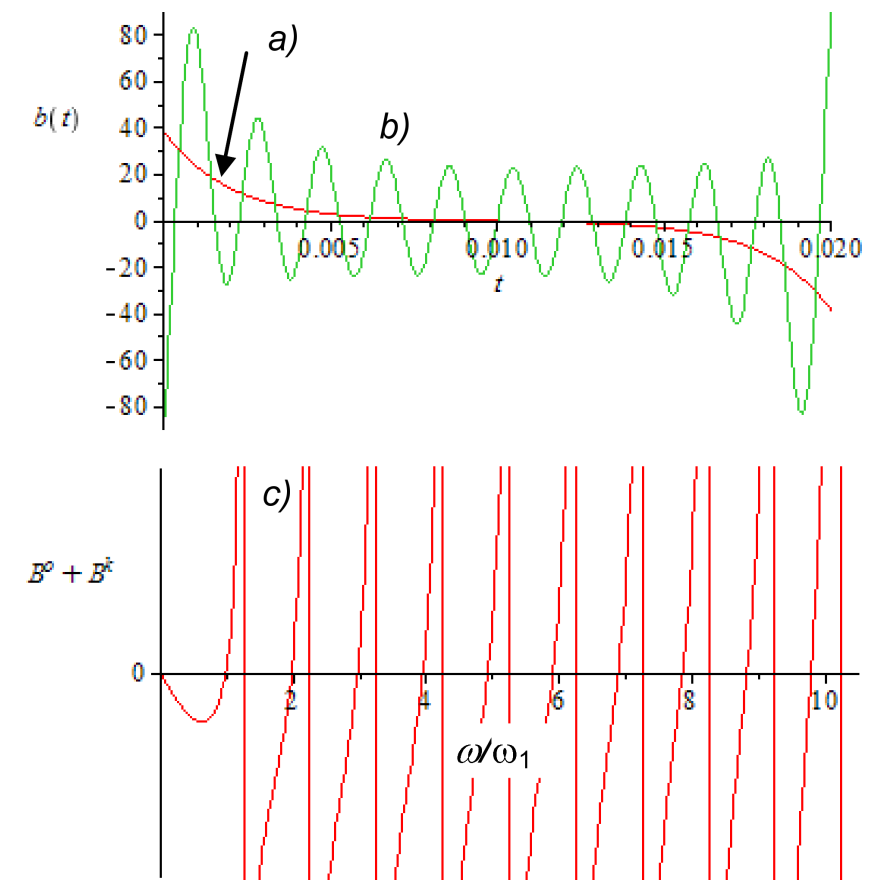

Fig. 5. In time-domain a) load $b^{o}(t)$, b) compensator $-b^{k}(t)$ and in frequency-domain c) $B^{o}\left(\omega / \omega_{1}\right)+B^{k}\left(\omega / \omega_{1}\right)$ for $\alpha=1 / 4$ 


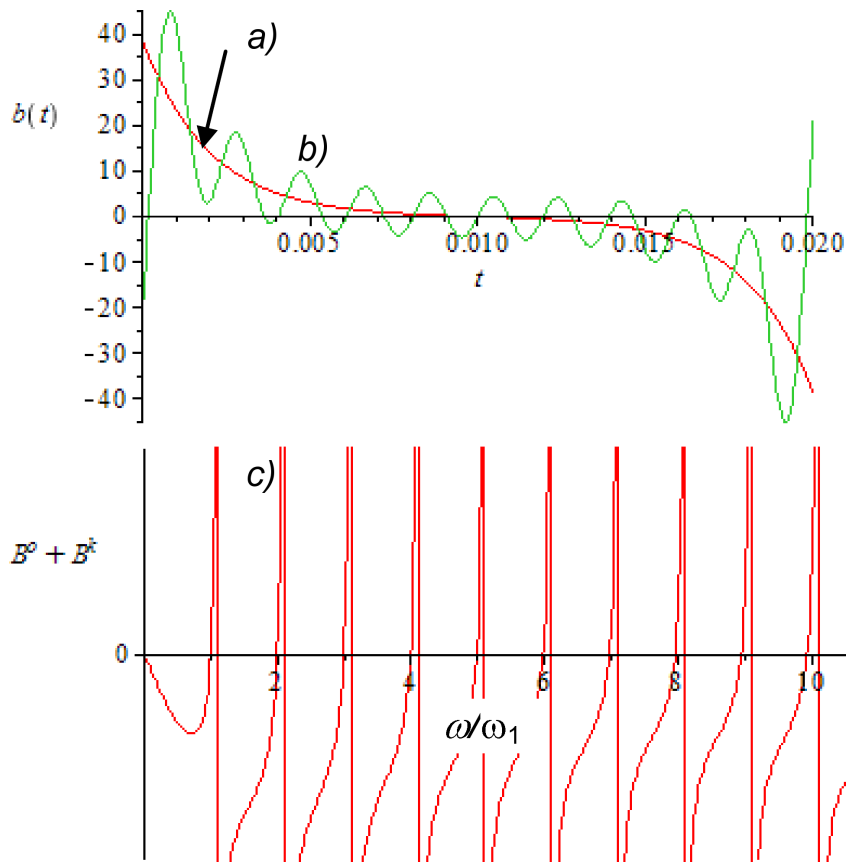

Fig. 6. In time-domain a) load $b^{o}(t)$, b) compensator $-b^{k}(t)$ and in frequency-domain c) $B^{o}\left(\omega / \omega_{1}\right)+B^{k}\left(\omega / \omega_{1}\right)$ for $\alpha=0.1$

\section{Conclusions}

The frequency response method which has been used until now to synthesis LC compensators and has been considered the only one [1], has its counterpart in a time-domain. In both approaches the LC parameters can be found with simple optimization techniques for a linear system.

\section{REFERENCES}

[1] L.S Czarnecki, "Discussion on a uniform concept of reactive power of nonsinusoidal currents in a time-domain", Electrical Review R 85 (6), CD-ROM, 2009.

[2] M. Siwczyński and M.Jaraczewski, "The $\mathrm{L}^{1}$-impulse method as an alternative to the Fourier series in the power theory of continues time systems". Bull. Pol. Ac.: Tech. 57 (1), 79-86 (2009).

[3] M. Siwczyński , "Decompositions: active current, scattered current, reactive current in time-domain - single-phase circuits", Electrical Review 6, 11-17 (2010).

[4] M. Pasko, "Choice of the two-terminal components, compensating reactive current component of the linear load fed with distorted voltage", SPETO'92 1, CD-ROM (1992).

[5] M. Pasko, "Improvement of the working conditions of the real distorted voltage sources using two-terminal LC components", Scientific Notebooks of the Silesian University of Technology: Electrotechnics 117, 45-62 (1991).

[6] M. Pasko and J. Walczak, Optimization of the Power Quality Coefficients of Electrical Circuits with Nonsinusoidal Periodic Signals, Publishing House of the Silesian University of Technology, Gliwice, 1996. 